\title{
Extracorporeal membrane oxygenation following pediatric cardiac surgery: development and outcomes from a single center experience
}

Hideshi Itoh $* \ddagger$, Shingo Ichiba $\uparrow$, Yoshihito Ujike $\ddagger$, Shingo Kasahara $*$, Sadahiko Arai $*$, Shunji Sano *

* Department of Cardiovascular Surgery, Okayama University Hospital

$\dagger$ Department of Community and Emergency Medicine, Okayama University Graduate School of Medicine, Dentistry and Pharmaceutical Sciences

\$ Department of Emergency and Critical Care Medicine, Okayama University Graduate School of Medicine, Dentistry and Pharmaceutical Sciences

Disclaimer: The authors hereby state that the article is original, presently not under consideration for publication in another journal and has not been published previously. If the manuscript entitled "Extracorporeal membrane oxygenation following pediatric cardiac surgery: development and outcomes from a single center experience" is published, the undersigned author(s) give(s) all copyright ownership to "Perfusion".

Disclosure: I declare on behalf of myself and all authors the following:

We have no material, financial, or other relationship with any healthcare-related business or other entity whose products or services may be discussed in, or directly affected in the marketplace by, this manuscript.

Corresponding Author: Hideshi Itoh

Department of Cardiovascular Surgery, Okayama University Hospital

2-5-1, Shikata, Okayama, 700-8558, Japan

E-Mail: grape@md.okayama-u.ac.jp

Tel: $+81-86235-7357$

Fax: $+81-86235-7431$

Running Head: ECMO after pediatric cardiac surgery 
Itoh, 2

\section{Key-words:}

Extracorporeal membrane oxygenation, congenital heart disease, cardiac surgery,

pediatric, hypoplastic left heart syndrome 
Itoh, 3

\begin{abstract}
Extracorporeal membrane oxygenation (ECMO) has emerged as an effective
\end{abstract}

mechanical support following cardiac surgery with respiratory and cardiac failure.

However, there are no clear indications for ECMO use after pediatric cardiac surgery.

We retrospectively reviewed medical records of 76 pediatric patients [mean age, 10.8

months (0-86); mean weight, $5.16 \mathrm{~kg}(1.16-16.5)]$ with congenital heart disease who

received ECMO following cardiac surgery between January 1997 and October 2010.

Forty-five patients were treated with aggressive ECMO approach (aggressive ECMO

group, April 2005-October 2010) and 31 with delayed ECMO approach (delayed

ECMO group, January 1997-March 2005). Demographics, diagnosis, operative

variables, ECMO indication, and duration of survivors and non-survivors were

compared. Thirty-four patients $(75.5 \%)$ were successfully weaned from ECMO in

aggressive ECMO, and 26 (57.7\%) were discharged. Conversely, eight patients (25.8\%)

were successfully weaned from ECMO in delayed ECMO, and two (6.5\%) were

discharged. Forty-five patients with shunted single ventricle physiology (aggressive: 29

patients, delayed: 16 patients) received ECMO, but only 15 (33.3\%) survived off and 
Itoh, 4

were discharged. Survival rate of aggressive ECMO was significantly better compared with delayed ECMO $(\mathrm{p}<0.01)$. Also, ECMO duration was significantly shorter among aggressive ECMO survivors $(96.5 \pm 62.9 \mathrm{~h}, P<0.01)$. Thus, aggressive ECMO approach is a superior strategy compared to delayed ECMO approach in pediatric cardiac patients. Aggressive ECMO approach improved our outcomes of neonatal and pediatric ECMO. 


\section{INTRODUCTION}

Extracorporeal membrane oxygenation (ECMO) has emerged as an effective mechanical support following cardiac surgery with respiratory and cardiac failure. In 1976, Bartlett et al. ${ }^{1}$ reported the successful use of ECMO for a neonatal patient with respiratory failure, and ECMO has since been used effectively for a variety of indications including preoperative hemodynamic support, low cardiac output after cardiopulmonary bypass (CPB), sudden cardiac arrest, and as a bridge to heart transplantation. ${ }^{2}$ The Extracorporeal Life Support Organization (ELSO) registry reports that the rates of survival off ECMO and survival to discharge among neonatal cardiac patients are $59 \%$ and $39 \%$, respectively; in pediatric patients, these values are $62 \%$ and $46 \%$, respectively. The rates of survival off ECMO and to discharge among neonates subject to extracorporeal cardiopulmonary resuscitation are $63 \%$ and $37 \%$, and in pediatric patients these rates are $52 \%$ and $38 \%$, respectively. ${ }^{3}$ Outcomes of ECMO have been developing and still have been keeping the improvement scope for better outcomes, especially in patients with congenital heart disease.

In our institute, we did not have any criteria for ECMO introduction until 
Itoh, 6

March 2005, but we changed our strategy of ECMO therapy and introduced an aggressive approach toward indications for ECMO from April 2005 wherein we did not hesitate to introduce ECMO. Before we had changed our strategy of ECMO therapy in April 2005, we had felt the negative image of ECMO because of our poor results of survival off ECMO in patients with congenital heart disease.

The purpose of this study was to evaluate the effects of this aggressive approach of ECMO initiation since we changed our strategy of ECMO compared with the previously followed delayed approach for introducing ECMO after pediatric cardiac surgery by reviewing our single center experiences. 


\section{MATERIALS AND METHODS}

We retrospectively reviewed medical records of 76 pediatric patients with congenital heart disease who received ECMO following cardiac surgery between January 1997 and October 2010 at Okayama University Hospital, Japan.

Two groups were compared those who were treated with the aggressive ECMO approach (45 patients from April 2005 to October 2010), and those were treated with the delayed ECMO approach (31 patients from January 1997 to March 2005). The aggressive ECMO approach was defined as commencement of ECMO as early as possible before end-organ dysfunction or complete circulatory collapse, and without hesitate to introduction of ECMO. The outcomes were categorized as follows: survived off ECMO (successful weaning from ECMO support), survival to hospital discharge, and survival during outpatient follow-up. Demographics, diagnosis, operative variables, and ECMO indication and duration of survivors and non-survivors were compared. We compared the lactate level of both groups before and 2 hours later introduced ECMO.

We defined the indications for aggressive ECMO as follows: mean arterial blood pressure less than $35 \mathrm{mmHg}$, anuria, high lactate level (greater than $5.0 \mathrm{mmol} / \mathrm{L}$ ) 
and acidosis with $\mathrm{pH}$ less than 7.3, hypoxia with arterial oxygen saturation less than $60 \%$ with $100 \% \mathrm{FiO}_{2}$ (fraction of inspired oxygen) on mechanical ventilatory support. ECMO was established via a median sternotomy, and cannulation of the ascending aorta or inominate artery for return, and drainage via a 3-mm polytetrafluoroethylene graft to the right atrium (in cases of after a Norwood stage 1 procedure). For the patients with a right ventricle $(\mathrm{RV})$ to pulmonary artery $(\mathrm{PA})$ shunt after a Norwood stage 1 procedure in hypoplastic left heart syndrome, we opened the RV-PA shunt and controlled the pulmonary blood flow by the degree of clipping the shunt.

We used a hollow fiber membrane oxygenator (Biocube ${ }^{\circledR}$; Nipro, Osaka, Japan) and a centrifugal pump (Gyro®; Kyocera, Kyoto, Japan) with a 6-mm heparin coated tube (Biomate ${ }^{\circledR}$; Toyobo, Osaka, Japan) for ECMO device. The initial ECMO flow rate was set at $150-180 \mathrm{ml} / \mathrm{kg} / \mathrm{min}$. Anticoagulation was accomplished by drip infusion of heparin sodium to maintain an activated clotting time of 150-200 seconds. Hemoglobin concentration was maintained above $10 \mathrm{~g} / \mathrm{dL}$ by transfusion of packed red blood cells during full support. We maintained the bladder temperature at $35^{\circ} \mathrm{C}$ and applied minimal ventilatory support during ECMO. The intrathoracic cavity was 
Itoh, 9

irrigated with sterile warmed normal saline every 3 days.

We tried to wean the patient off ECMO when the lactate level was less than

$2.0 \mathrm{mmol} / \mathrm{L}$, the urine output was increased to equal or greater than $1.0 \mathrm{ml} / \mathrm{kg} / \mathrm{h}$ for as

long as $24 \mathrm{~h}$, and the arterial pulse pressure was increased to equal or greater than 10

mmHg. We started inotropic agents for $6 \mathrm{~h}$ and gradually reduced the ECMO flow rate

under stable hemodynamic conditions. We weaned the patient off ECMO within $12 \mathrm{~h}$.

Descriptive statistics are expressed as mean \pm standard deviation $(s)$. The

$\chi^{2}$ test for a $(2 \times N)$ table, Kruskal-Wallis test and student $t$ test were used to evaluate differences between groups for statistical significance. A $P$-value of $<0.05$ was considered to have statistical significance. 


\section{RESULTS}

The mean age of all patients was 10.8 months (range, 0 days -86 months) and the mean weight was $5.16 \mathrm{~kg}(1.16-16.5 \mathrm{~kg})$. The demographics data of both aggressive ECMO and delayed ECMO was shown in Table 1. Cardiopulmonary bypass time (aggressive: $187.20 \pm 117.48$ min, delayed: $194.90 \pm 115.33 ; \mathrm{P}=0.400$ ) and aortic cross clamp (aggressive: $79.93 \pm 44.01 \mathrm{~min}$, delayed: $64.62 \pm 44.78 \mathrm{~min} ; \mathrm{P}=0.105$ ) time during surgery were not significantly difference. Thirty-four patients (75.5\%) from aggressive ECMO $(n=45)$ survived off ECMO and 26 patients $(57.7 \%)$ survived to hospital discharge (Table 2). Eight patients (25.8\%) from delayed ECMO $(\mathrm{n}=31)$ survived off ECMO and 2 patients (6.5\%) survived to hospital discharge (Table 2). Thirty-one patients $(31 / 76,40.8 \%)$ had biventricular physiology and 45 patients $(45 / 76$, 59.2\%) single ventricle physiology. All the patients with single ventricle physiology treated with delayed ECMO died in the hospital (Table 3). The patients with biventricular physiology treated with the aggressive ECMO had significantly better results than the other groups. The patients who survived off ECMO and hospital discharge following the aggressive ECMO had significantly shorter ECMO duration 
Itoh, 11

than those following delayed ECMO (Table 4).

The timing of introduction is shown in Table 5. When ECMO was indicated for patients in the intensive care unit (ICU), aggressive ECMO group had a significantly shorter duration of ECMO days after operation than that of delayed ECMO group.

Our indication criteria for ECMO are shown in Table 5. In aggressive ECMO group, low cardiac out put syndrome was the significant reason for indication. In delayed ECMO group, cardiac arrest and hypoxia were the two major reasons for ECMO.

The lactate level of before and after introduced ECMO was shown in Table 6. The lactate level of both groups was not significantly difference. 
Itoh, 12

\section{DISCUSSION}

ECMO support following pediatric cardiac surgery provides effective support

for postoperative cardiac and pulmonary dysfunction refractory to conventional medical management. ${ }^{4}$ ECMO is an advanced therapy for acute cardiac and/or respiratory failure associated with congenital heart disease and pulmonary disease. ${ }^{5}$ Indications for ECMO are affected by many factors, including ventricular function, magnitude of conventional inotropic support, and pulmonary function. No standard indication criteria or management guidelines have been established for ECMO in congenital heart disease because of its complex nature and specificity of use. ${ }^{6}$

At our institution, in April 2005, the approach for ECMO was changed from a delayed ECMO approach where patients were managed without ECMO as long as possible to an aggressive ECMO approach where patients were indicated for ECMO as early as possible before a catastrophic event such as cardiac arrest occurred. In our results, the aggressive ECMO approach revealed a significantly better result in terms of ECMO survival and survival to hospital discharge than delayed ECMO group.

Ungeleider et al. have suggested that mechanical assistance should be routine 
after Norwood staged 1 procedure, and described aggressive approach of mechanical assistance improved hospital survival rate. ${ }^{7}$ Cooper et al. have suggested that initiation of ECMO support should be based on "urgent" rather than "emergency" criteria, i.e., before the occurrence of end-organ dysfunction or circulatory collapse. ${ }^{8}$ The risk factors for mortality due to ECMO are as follows: age below 1 month, male gender, long duration of mechanical ventilation support prior to introducing ECMO, and development of renal or hepatic dysfunction during ECMO. ${ }^{6,9}$ Following pediatric cardiac surgery, it is critical to maintain both systemic and pulmonary blood flow. The advantage of aggressive ECMO is prevention of ventilator-induced lung injury caused by respiratory care injuries resulting from setting up mechanical ventilation.

Booth et al. demonstrated that the survival of cardiac patients supported by ECMO is associated with indication and cardiac diagnosis. ${ }^{2}$ The ELSO registry reported that rates of survival to weaning from ECMO and survival to hospital discharge were $59 \%$ and $39 \%$ in neonatal patients, and $62 \%$ and $46 \%$ in pediatric patients, respectively. ${ }^{3}$ In our study, rates of survival to weaning from ECMO and survival to hospital discharge were $75.5 \%$ and $57.7 \%$, respectively. Our aggressive ECMO 
approach was shown to be superior to that of the ELSO registry average. Hence, the aggressive ECMO approach may result in better ECMO outcomes for patients with congenital heart disease than delayed ECMO approach.

Patients with single ventricle physiology, especially those with hypoplastic left heart syndrome, had a significantly higher mortality rate than those with biventricular physiology. ${ }^{6,10}$ In our review, aggressive ECMO approach brings better outcomes at least patients with single ventricle physiology than delayed ECMO approach.

It is well known that long ECMO duration increases the risk of complications such as bleeding, hemolysis, and systemic inflammatory syndrome. Long ECMO duration also increases the mortality rate associated with ECMO, and may affect the progression of multiple organ dysfunction and have a negative influence on immunological systems. ${ }^{6,10,12,13}$ A previous study of ours as well as one by Baslaim et al. clearly indicated that patients with long ECMO duration (more than 3 days) may benefit less from ECMO support and may have an increased risk of mortality. ${ }^{6,10}$ In general, the purpose of pediatric ECMO as a mechanical circulatory 
support following cardiac surgery is recovery of cardiac function by unloading the right and left ventricle preloads and increasing the ECMO flow rate as well as resting the lungs from the high demand for oxygen saturated blood by the body. On the other hand, increasing the ECMO flow rate increases the left ventricular afterload and wall stress. These ECMO mismatches have a negative impact and outcome during pediatric ECMO following cardiac surgery. Hence, not only early introduction of ECMO but also early weaning as early as possible from ECMO might help to get better outcomes.

Several groups have reported improved survival in patients placed on ECMO in the operating room compared with those cannulated in the ICU. ${ }^{12,13}$ Chaturvedi reported avoidance of severe end-organ damage before restoration of adequate perfusion to pursue an aggressive approach for early indication of ECMO with the aim of reducing the morbidity and mortality associated with prolonged periods of hypoperfusion and cardiac arrest. ${ }^{13}$ Our review also showed that it might be better to choose an aggressive ECMO approach of early introduction of ECMO before progress to multiple organ dysfunctions could possibly prevent cardiac arrest, which results a poor outcome. The ELSO registry report in 2009 showed a poor outcome for 
Itoh, 16

extracorporeal cardiopulmonary resuscitation after cardiac arrest, with survival rates of $26 \%$ in neonates, $47 \%$ in infants, and $39 \%$ in pediatric patients. ${ }^{3}$

In conclusion, we recommend an aggressive ECMO approach following pediatric cardiac surgery, which requires early introduction and early discontinuation of ECMO support before end-organ dysfunction and circulatory collapse, rather than the conventional approach of delaying ECMO introduction. Aggressive ECMO approach improved outcomes of ECMO therapy in our institution. 


\section{REFERENCES}

1. Bartlett RH, Gazzaniga AB, Jefferies MR, et al. Extracorporeal membrane oxygenation (ECMO) cardiopulmonary support in infancy. Trans Am Soc Artif Intern Organs. 1976;22: 80-93.

2. Booth KL, Roth SJ, Laussen PC, et al. Extracorporeal membrane oxygenation support of the Fontan and bidirectional Glenn circulations. Ann Thorac Surg. 2004;77: 1341-1348,

3. ECLS Registry Report. International summary, July 2009. Extracorporeal life support organization. Ann Arbor (MI).

4. Laforte A, Walter EM D, Hetzer R, et al. Extracorporeal membrane oxygenation for intraoperative cardiac support in children with congenital heart disease. Interact Cardio Vasc Thorac Surg. 2010;10: 753-758.

5. Schmid C, Philipp A, Mueller T, Hilker M. Extracorporeal life support systems, indications, and limitations. Thorac Cardiovasc Surg. 2009.57: 449-454.

6. Itoh H, Kasahara S, Sano S, et al. Risk factor analysis of cardiac ECMO for congenital heart disease. Membrane Oxygenator (Japanese). 2009;32: 43-46. 
7. Ungerleider RM, Shen I, Burch G, et al. Use of routine ventricular assist following the first stage Norwood procedure. Cardiol Young. Feb 2004; 14 Suppl 1:61-64.

8. Cooper DS, Jacobs JP, Ravishankar, et al. Cardiac extracorporeal life support:state of the art in 2007. Cardiol Young. 2007;17: 104-115.

9. Morris M, Ittenback R, Godinez R, et al. Risk factors for mortality in 137 pediatric cardiac intensive care unit patients managed with extracorporeal membrane oxygenation. Crit Care Med. 2004;32: 1061-1069.

10. Baslaim G, Basshore J, Jamjoom A, et al. Can the outcome of pediatric extracorporeal membrane oxygenation after cardiac surgery be predicted? Ann Thorac Surg. 2006;12: 21-27.

11. Kumer S, Zurakowski D, Moulick A et al. Extracorporeal membrane oxygenation in postcardiotomy patients: factors influencing outcome. J Thorac Cardiovasc Surg. 2010;140: 330-336.

12. Kolovos NS, Bratton SL, Moler FW, et al. Outcome of pediatric patients treated with extracorporeal life support after cardiac surgery. Ann Thorac Surg. 2003;76: 1435-1441. 
Itoh, 19

13. Chaturvedi RR, Marcrae D, Brown KL, et al. Cardiac ECMO for biventricular hearts after pediatric open heart surgery. Heart. 2004;90: 545-551. 
Itoh, 20

\section{Figure Legend}

Table 1: The demographics data of aggressive and delayed ECMO groups

AS, aortic stenosis; DORV, double outlet right ventricule; TOF, tetralogy of fallot; PA, pulmonary atrisia; VSD, ventricular septal defect; TAPVC, total anomalous pulmonary venous connection; CoA, coactation of aorta; BWG, Bland-White-Garland; HLHS, hypoplastic left heart syndrome; SA, single atrium; SV, single ventricle; TA, tricuspid atrisia; DILV, double inlet left ventricule; PA, pulmonary atrisia; IVS, intact ventricular septum; IAA, interrupted aortic arch; MS, mitral stenosis; AVSD, atrioventricular septal defect; TGA, transposition of great arteries; PTA, persistent truncus arteriosus

Table 2: Comparison of Extracorporeal membrane oxygenation (ECMO)

decannulation and hospital discharge ratio

Table 3: Comparison of Patients with Biventricular and Single ventricle physiology

Table 4: Comparison of ECMO duration $($ mean $\pm s)$

\section{Table 5: Patients Profiles}

This table shows the comparison between groups $\mathrm{A}$ and $\mathrm{B}$ in terms of time to 
Itoh, 21

introduction of ECMO and indication criteria for ECMO.

OR: operating room; ICU: intensive care unit; LCOS: low cardiac output syndrome;

n.s.: non-significant difference. Numbers inside () indicate the number of patients.

Table 6: The lactate level of before and after ECMO 
Table 1.

\begin{tabular}{|c|c|c|c|}
\hline \multicolumn{4}{|c|}{ Aggressive ECMO $(n=45)$} \\
\hline \multicolumn{2}{|l|}{ Biventricle $(n=16)$} & \multicolumn{2}{|c|}{ Singleventricle $(n=29)$} \\
\hline Critical AS & 4 & HLHS & 9 \\
\hline DORV & 3 & HLHS variant & 7 \\
\hline TOF & 3 & $\mathrm{SA} / \mathrm{SV}$ & 5 \\
\hline $\mathrm{PA} / \mathrm{VSD}$ & 2 & $\mathrm{TA}$ & 3 \\
\hline TAPVC & 2 & DILV & 2 \\
\hline $\mathrm{CoA} / \mathrm{VSD}$ & 1 & PA/IVS & 1 \\
\hline \multirow[t]{2}{*}{ BWG syndrome } & 1 & IAA & 1 \\
\hline & & Congenital MS & 1 \\
\hline \multicolumn{4}{|c|}{ Delayed ECMO ( $\mathrm{n}=31)$} \\
\hline \multicolumn{2}{|l|}{ Biventricle $(n=15)$} & \multicolumn{2}{|c|}{ Singleventricle $(n=16)$} \\
\hline DORV & 3 & HLHS & 5 \\
\hline PA/VSD & 3 & HLHS variant & 3 \\
\hline AVSD & 2 & PA/IVS & 3 \\
\hline $\mathrm{TOF}$ & 2 & DILV & 2 \\
\hline TAPVC & 1 & $\mathrm{SA} / \mathrm{SV}$ & 2 \\
\hline TGA & 1 & TA & 1 \\
\hline Critical AS & 1 & & \\
\hline PTA & 1 & & \\
\hline Myocarditis & 1 & & \\
\hline
\end{tabular}

